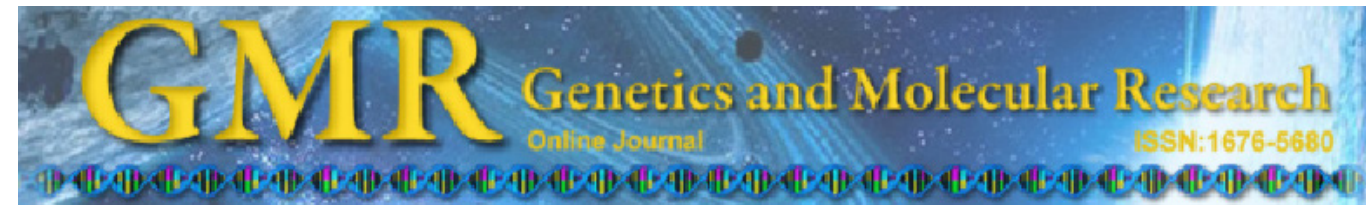

\title{
Somatic copy-neutral loss of heterozygosity and copy number abnormalities in Malaysian sporadic colorectal carcinoma patients
}

\author{
Y.Y. Yam ${ }^{1}$, B.P. Hoh ${ }^{1,2}$, N.H. Othman ${ }^{3,4}$, S. Hassan ${ }^{5}$, M.M. Yahya ${ }^{5}$, \\ Z. Zakaria ${ }^{5}$ and R. Ankathil ${ }^{1}$ \\ ${ }^{1}$ Human Genome Centre, School of Medical Sciences, \\ Universiti Sains Malaysia, Health Campus, Kubang Kerian, \\ Kelantan, Malaysia \\ ${ }^{2}$ Institute for Medical Molecular Biotechnology, Faculty of Medicine, \\ Universiti Teknology MARA, Sungai Buloh Campus, Selangor, Malaysia \\ ${ }^{3}$ Clinical Research Platform, Universiti Sains Malaysia, Health Campus, \\ Kubang Kerian, Kelantan, Malaysia \\ ${ }^{4}$ Department of Pathology, School of Medical Sciences, \\ Universiti Sains Malaysia, Health Campus, Kubang Kerian, \\ Kelantan, Malaysia \\ ${ }^{5}$ Department of Surgery, School of Medical Sciences, \\ Universiti Sains Malaysia, Health Campus, Kubang Kerian, \\ Kelantan, Malaysia \\ Corresponding author: R. Ankathil \\ E-mail: rankathil@hotmail.com
}

Genet. Mol. Res. 12 (1): 319-327 (2013)

Received January 19, 2012

Accepted September 3, 2012

Published February 7, 2013

DOI http://dx.doi.org/10.4238/2013.February.7.1

ABSTRACT. Colorectal cancer is one of the most common cancers in many countries, including Malaysia. The accumulation of genomic alterations is an important feature of colorectal carcinogenesis. A better understanding of the molecular events underlying the stages of colorectal carcinogenesis might be helpful in the detection and management of the disease. We used a commercially available singlenucleotide polymorphism genotyping array to detect both copy number 
abnormalities (CNAs) and copy-neutral loss of heterozygosity (LOH) in sporadic colorectal carcinomas. Matched tumor and normal tissues of 13 colorectal carcinomas (Dukes' stages A-D) were analyzed using a $250 \mathrm{~K}$ single nucleotide polymorphism array. An additional assay was performed to determine the microsatellite instability status by using the National Cancer Institute-recommended BAT-26 panel. In general, copy number gain $(92.3 \%)$ was most common, followed by copy number loss $(53.8 \%)$ and copy-neutral LOH (46.2\%). Frequent CNAs of gains and losses were observed on chromosomes $7 \mathrm{p}, 8,13 \mathrm{q}, 17 \mathrm{p}, 18 \mathrm{q}$, and 20q, and copy-neutral LOH was observed on chromosomes 2, 6, 12, 13q, 14q, 17, 20p, 19q, and 22q. Even though genomic alterations are associated with colorectal cancer progression, our results showed that DNA CNAs and copy-neutral LOH do not reflect disease progression in at least $50 \%$ tumors. Copy-neutral $\mathrm{LOH}$ was observed in both early and advanced tumors, which favors the involvement of these genomic alterations in the early stages of tumor development.

Key words: Sporadic colorectal cancer; Copy number abnormality; Copy-neutral loss of heterozygosity

\section{INTRODUCTION}

Colorectal cancer (CRC) is a common cancer in many developed countries and is also one of the most common cancers in Malaysia. In the Malaysian National Cancer Registry (2006), CRC ranked as the 2nd most frequently occurring cancer among both genders. The commonly understood mechanism is familial CRC, which progresses through a series of well-defined steps, including early and late adenomas, invasive cancer, and eventually metastatic lesions (Fearon and Vogelstein, 1990). The less understood mechanism is sporadic CRC. Although significant data have been generated regarding genomic events and the associated genetic instability that accompanies various stages of tumorigenesis, significant gaps remain. The acquisition of genomic alterations is generally known to play a significant role in the progression of sporadic CRC (Stratton et al., 2009). These somatic genetic alterations include point mutations, small insertion/deletion events, translocations, copy number $(\mathrm{CN})$ changes, and loss of heterozygosity (LOH), which eventually attenuate gene expression. A better understanding of the molecular events underlying the various stages of CRC may help in the detection and prevention of premalignant lesions.

Genome-wide analyses of chromosomal CN abnormalities (CNAs) have provided insight on CRC carcinogenesis, including defining the genomic events that occur during various stages, although the results are inconsistent (Aragane et al., 2001; Choi et al., 2002; He et al., 2003; Knosel et al., 2004; Diep et al., 2006; Sheffer et al., 2009; Lagerstedt et al., 2010; Poulogiannis et al., 2010; Lin et al., 2011). However, reports on the occurrence of copy-neutral LOH have been scant.

We used single nucleotide polymorphism (SNP) microarray to investigate both CNAs and copy-neutral LOH in tumor tissues of sporadic CRC patients, encompassing tumors ranging from Dukes' A to Dukes' D. 


\section{MATERIAL AND METHODS}

\section{Samples}

Samples were obtained from patients with histologically confirmed CRC who volunteered for the study. The cancers were surgically resected. Patients with a family history of CRC or other cancers and who had undergone neo-adjuvant therapy were excluded. This study was approved by the Research Review Board and Human Ethics Committee of Universiti Sains Malaysia and the Malaysian Ministry of Health (NMRR ID: 4621).

Cancerous areas were examined in a fresh state with the help of pathologists, and portions of tumors (approximately $4 \times 4 \mathrm{~mm}$ ) were removed and immediately stored in a freezer at $-80^{\circ} \mathrm{C}$ until further analysis. Portions of the epithelial layer from tumor-free surgical margins were also sampled and treated in the same way. The rest of the cancer tissues was subjected to routine histopathological examination.

\section{DNA extraction}

The frozen tissues were subjected to DNA extraction. Tissues were lysed in ATL Buffer (QIAamp DNA Micro Kit, QIAGEN, Hilden, Germany) and digested overnight (approximately $16 \mathrm{~h}$ ) with proteinase $\mathrm{K}$ at $56^{\circ} \mathrm{C}$. An additional $20 \mu \mathrm{L} 20 \mathrm{mg} / \mathrm{mL}$ proteinase $\mathrm{K}$ was spiked in on the 2 nd day to ensure complete digestion. The subsequent steps were carried out according to manufacturer instructions for the DNA extraction kit. The extracted double-stranded DNA was quantified using a Tecan Infinite ${ }^{\circledR} 200$ NanoQuant (Tecan Group Ltd., Männedorf, Switzerland) at an optical density (OD) of $260 \mathrm{~nm}$. The integrity of the DNA was checked on 1\% agarose gel. For samples with intact DNA and OD 260/280 ratios between 1.8 and 2.0, we carried out microarray procedures according to manufacturer instructions on an Affymetrix 500K GeneChip Manual (Affymetrix Inc., Santa Clara, CA, USA) with slight modification for the purification of PCR products, which was performed with Agencourt AMPure (Agencourt Bioscience Corp., Beverly, MA, USA).

\section{Sample preparation for GeneChip Human Mapping 250K SNP array}

Microarray procedures for tumor and normal DNA from each case were run in the same batch to reduce technical variation. The mean OD 260/280 ratios of extracted DNA selected for microarray were 1.9. Tumor and normal DNAs were digested with NspI restriction enzyme (New England Biolabs Inc., Ipswich, MA, USA). The digested DNA was ligated to a universal NspI adaptor (obtained from the microarray manufacturer) using T4 Ligase (New England Biolabs Inc.) followed by PCR using universal primers that recognized the sequence of the NspI adaptor. Four replicates of the PCR products from the same sample were pooled, purified, and normalized to $2 \mu \mathrm{g} / \mu \mathrm{L}$ before fragmentation with DNAse I (obtained from the microarray manufacturer) and labeling with biotin. Tumor and normal samples were each hybridized to Affymetrix GeneChip Human Mapping $250 \mathrm{~K}$ Nsp arrays for $17 \mathrm{~h}$ at $49^{\circ} \mathrm{C}$. The hybridized arrays were washed and stained using a Fluidics Station 450 (Affymetrix) and scanned with a GeneChip Scanner 3000 7G (Affymetrix). Scanned images of each sample were generated with Affymetrix GeneChip Operating System. 


\section{Data analysis}

Scanned images (raw intensity data) of each sample were imported into Affymetrix Genotyping Console 4.0 to generate a genotyping call at a dynamic model threshold of 0.17 . Quantile normalization at the probe level was performed, followed by paired analysis for $\mathrm{CN}$ and $\mathrm{LOH}$. The transition decay for inferring both $\mathrm{CN}$ and $\mathrm{LOH}$ was set at $1 \mathrm{Mb}$. In paired analysis, $\mathrm{CN}$ gain or loss was determined by comparing the intensity of matched tumor and normal DNA. A CN state of 2 indicated normal CN, 0 and 1 indicated $\mathrm{CN}$ loss, and $\mathrm{CN}$ gain was indicated by states of 3 and 4 .

$\mathrm{LOH}$ was identified if a heterozygous call in normal DNA was detected as a homozygous call in tumor DNA. Copy-neutral LOH was determined by simultaneous analysis of both $\mathrm{CN}$ and $\mathrm{LOH}$ data - a region of $\mathrm{LOH}$ with a $\mathrm{CN}$ state of 2. Paired analysis for $\mathrm{CN}$ and LOH was also performed using Copy Number Analyzer for GeneChip 3.0 (Nannya et al., 2005).

\section{RESULTS}

\section{Demographic and clinicopathological information}

A total of 13 sporadic CRC cases were included in the study. The majority of the cancers $(69 \%)$ were in advanced stages (Table 1$)$. The tumors sampled were mainly from rectum $(84.6 \%)$; the rest were from sigmoid, which reduced the heterogeneity to some extent. The age of patients ranged from 40 to 90 years with a mean age of 62 years. The patients included 11 of Malay ethnicity and one each of Chinese and Siamese ethnicity. Twelve of the tumors were classical adenocarcinoma and one was mucinous adenocarcinoma. All cancers were of moderate grade of differentiation.

Table 1. Distribution of whole genomic alterations in the colorectal cancer tissues.

\begin{tabular}{|c|c|c|c|c|c|c|c|}
\hline \multirow[t]{2}{*}{$\begin{array}{l}\text { Sample } \\
\text { ID }\end{array}$} & \multirow[t]{2}{*}{ Gender } & \multirow[t]{2}{*}{$\begin{array}{l}\text { Age } \\
\text { (years) }\end{array}$} & \multirow[t]{2}{*}{ Tumor site } & \multirow[t]{2}{*}{$\begin{array}{c}\text { Cancer staging } \\
\text { (Dukes' classification) }\end{array}$} & \multicolumn{2}{|c|}{$\begin{array}{l}\text { Chromosomes/regions involved } \\
\text { in copy number abnormalities }\end{array}$} & \multirow[t]{2}{*}{$\begin{array}{l}\text { Chromosomes involved } \\
\text { in copy-neutral LOH }\end{array}$} \\
\hline & & & & & Loss & Gain & \\
\hline 01 & M & 55 & Rectum & $\mathrm{D}$ & - & $20 q$ & - \\
\hline 02 & M & 69 & Sigmoid & $\mathrm{D}$ & - & $1 \mathrm{q}$ & - \\
\hline 03 & M & 53 & Rectum & $\mathrm{D}$ & $8 \mathrm{p}^{\mathrm{a}}, 17 \mathrm{p}, 18,21 \mathrm{q}^{\mathrm{b}}$ & $5 \mathrm{p}, 7 \mathrm{q}, 8 \mathrm{q}, 20 \mathrm{p}, 20 \mathrm{q}$ & $19 \mathrm{q}$ \\
\hline 04 & $\mathrm{~F}$ & 57 & Rectum & $\mathrm{D}$ & $8 \mathrm{p}, 14 \mathrm{q}, 17 \mathrm{p}, 18$ & $7 \mathrm{p}, 13 \mathrm{q}, 20 \mathrm{q}, \mathrm{X}$ & 20 pter-p12.1, 22q \\
\hline 05 & $\mathrm{~F}$ & 63 & Rectum & $\mathrm{B}$ & - & - & - \\
\hline 06 & $\mathrm{~F}$ & 53 & Sigmoid & $\mathrm{D}$ & 18 & $1 \mathrm{q}, 7 \mathrm{p}, 7 \mathrm{q}, 8 \mathrm{p}, 8 \mathrm{q}, 20 \mathrm{p}^{\mathrm{d}}, 20 \mathrm{q}$ & $2,12,13 \mathrm{q}, 17,22 \mathrm{q}^{\mathrm{c}}$ \\
\hline 07 & $\mathrm{~F}$ & 61 & Rectum & $\mathrm{D}$ & - & $5 \mathrm{p}$ & 6 pter-6p 21.33 \\
\hline 08 & M & 63 & Rectum & $\mathrm{C}$ & $8 \mathrm{p}, 17 \mathrm{p}, 18,20 \mathrm{p}$ & $7 \mathrm{p}, 8 \mathrm{q} 13.1$-qter, 13q, 20q, Xp & $14 \mathrm{q}$ \\
\hline 09 & $\mathrm{~F}$ & 72 & Rectum & A & - & $13 q$ & - \\
\hline 10 & $\mathrm{~F}$ & 62 & Rectum & A & - & $7 \mathrm{p}, 9,13 \mathrm{q}, 20 \mathrm{q}$ & $6,12,20 \mathrm{p}$ \\
\hline 11 & $\mathrm{~F}$ & 69 & Rectum & A & $8 \mathrm{p}^{\mathrm{a}}, 12 \mathrm{p}, 17 \mathrm{p}, 18$ & $6,7 \mathrm{p}, 7 \mathrm{q}, 8 \mathrm{q}, 19 \mathrm{q} 13.11$-qter, 20p, 20q & - \\
\hline 12 & M & 90 & Rectum & $\mathrm{D}$ & $8 \mathrm{p}, 17 \mathrm{p}$ & $9,13 q$ & - \\
\hline 13 & $\mathrm{~F}$ & 42 & Rectum & $\mathrm{C}$ & 18 & $5 \mathrm{p}, 13 \mathrm{q}, 16,20 \mathrm{p}, 20 \mathrm{q}$ & - \\
\hline
\end{tabular}

$\mathrm{F}=$ female; $\mathrm{M}=$ male. ${ }^{\mathrm{a}}$ Transition to copy number gain state for chromosome $8 \mathrm{p}$ was observed between chromosome 8p11.23-8p12 bands. ${ }^{b}$ Except 21q22.11-12 and 21q22.3. ${ }^{\text {EExcept } 22 q 13.3 . ~}{ }^{\mathrm{d}}$ Except a focal region in 20p12.1, which was observed to have copy number loss. $\mathrm{LOH}=$ loss of heterozygosity. 


\section{Genomic abnormality parameters}

All 13 tumors had different genomic abnormality profiles (see Table 1). Twelve tumors had at least one chromosomal arm (large scale) affected with CNAs. More CN gain than $\mathrm{CN}$ loss events were observed. Twelve of the 13 tumors (92.3\%) exhibited large-scale CN gains, whereas $\mathrm{CN}$ losses were observed in 7 of 13 tumors (53.8\%).

Recurrent CN gains were observed on chromosomes 20q (61.5\%), 13q (46.2\%), $7 p$ (38.5\%), 8q (30.8\%), and 20p (30.8\%), and recurrent CN loss occurred on chromosomes 18 (46.2\%), 8p (38.5\%), and 17p (38.5\%). Regions on chromosomes 1q, 5p, and 9 were less frequently involved $(\geq 10 \%$ but $<30 \%)$ in $\mathrm{CN}$ gains.

Compared to the frequency of CNAs, copy-neutral LOH frequency was lower and observed in 6 of 13 tumors (46.2\%). Two exceptions were tumors ID 10 and ID 06, in which copy-neutral $\mathrm{LOH}$ was higher than $\mathrm{CN}$ loss. The total copy-neutral $\mathrm{LOH}$ events ranged from 1 to 5 in the affected tumors. Two samples each had copy-neutral LOH of chromosomes $6 \mathrm{p}$ (ID 07 and ID 10), 12 (ID 06 and ID 10), 20p (ID 04 and ID 10), and 22q (ID 04 and ID 06).

\section{Genomic abnormality parameters according to early and advanced stages}

Based on stages, the tumors were divided into 2 groups, to examine further whether a distinct pattern of genomic abnormalities occurred between the 2 that might be important in various phases of disease progression. The 2 groups were Dukes' A and B as early cases and Dukes' $C$ and D as advanced cases. CN gains were observed in all (9/9) advanced CRC cases and in 75\% (3/4) of early CRC cases, whereas CN loss was observed in $66.7 \%$ (6/9) of advanced cases and in only $25 \%$ (1/4) of early cases. Among the CNAs, CN gains involving chromosomes $1 \mathrm{q}$ and $5 \mathrm{p}$ were observed only in the advanced-stage group. CN gains involving chromosomes $7 \mathrm{p}, 13 \mathrm{q}$, and $20 \mathrm{q}$ were the most commonly occurring events encountered in both groups. Furthermore, 55.6\% (5/9) of advanced cases and 25\% (1/4) of early cases displayed copy-neutral $\mathrm{LOH}$.

In almost half of the cases in the 2 groups, the total CNA and copy-neutral LOH events did not seem to increase as the disease progressed, and early stage tumors did not necessarily carry fewer CNAs. This phenomenon was seen predominantly in 3 advanced cases (ID 01, ID 02, and ID 07; all Dukes' D), which had no more than 2 large-scale genomic abnormality events. Conversely, at least 7 genomic abnormality events were observed in 2 early cases (ID 10 and ID 11; both Dukes' A). An additional assay using the BAT-26 marker, which determines the high microsatellite instability (MSI-H) status of the tumors (Nakao et al., 2004), indicated that only one advanced case (ID 07) was an MSI-H tumor.

\section{DISCUSSION}

In our study, genomic heterogeneity was observed among 13 samples, which showed various genomic abnormalities. The evaluation of the number of genomic abnormalities in individual tumors showed that a higher number did not reflect disease advancement and vice versa. We observed 3 advanced tumors that had fewer than 3 CNAs. An additional assay was performed to assess whether any of the tumors were MSI-H tumors. An MSI-H tumor is 
typically near diploid, but we found that this factor accounted for only 1 of 3 advanced-stage tumors, which had no more than 2 genomic abnormalities. We next compared the early stage and advanced-stage groups for distinct CNAs, and noted that recurrent gain involving chromosomes $1 \mathrm{q}$ and $5 \mathrm{p}$ occurred only in the advanced-stage group. Copy-neutral LOH events, in general, occurred less frequently than CNAs did, with 6 of $13(\sim 50 \%)$ samples showing copy-neutral LOH compared to 12 of 13 (92.3\%) showing the latter. Nonetheless, recurrent copy-neutral LOH was identified on chromosomes 6p, 12, 20p, and 22q.

Researchers have developed genetic models depicting the accumulation of DNA CN changes in disease progression (Knosel et al., 2004; Diep et al., 2006). A well-defined study that examined genomic changes in various stages of CRC has also shown that genomic changes increase significantly from 4 (Dukes'A) to 16\% (Dukes' D) of the entire genome (Lagerstedt et al., 2010). We were expecting advanced-stage samples to have a higher frequency of genomic changes compared with that in early stage tumors; however, approximately $50 \%$ of the tumors did not reflect this expectation. We also found that the magnitude of genomic change varied greatly even within tumors at the same stage. One of the Dukes' A samples had only one CNA, whereas another had up to 11 gross CNAs. In addition, the SNP array allowed assessment of copy-neutral LOH without bias toward any chromosome, a phenomenon that the majority of studies do not reflect or have overlooked.

Our results revealed up to 3 copy-neutral LOH events in yet another Dukes' A sample. Copy-neutral LOH was also observed in $55.6 \%$ of the advanced tumors. Copy-neutral LOH has been observed in previous studies that have used SNP array techniques (Andersen et al., 2007; van Puijenbroek et al., 2008). However, whether this event occurs at the earlier or later stages has not been indicated. This information hints at the role of candidate genes in disease processes. Having observed both early and advanced tumors in the present study, we suggest that copy-neutral LOH occurs quite early in CRC tumorigenesis and may be involved in CRC development.

Although the magnitude of genomic changes among the tumors varied greatly, common genomic CNAs were observed when individual chromosomes were considered. CNAs are often reported as overall frequencies to denote whether the event is random or non-random. Common chromosomal aberrations denote a non-random change and signify regions harboring key genes that drive tumorigenesis. We first observed that the common chromosomal aberrations are in concordance with previously published findings (Nakao et al., 2004; Staub et al., 2006; Ashktorab et al., 2010). However, the frequency of aberrations varies at least 2-fold compared with those in other studies (Table 2). For the results observed for chromosomes 13q, $17 \mathrm{p}$, and $18 \mathrm{p}$, a prominent difference between our study and a study from a neighboring country was noticed (Poeaim et al., 2005): our sample had a much higher frequency of alterations. For chromosomes $3 q$ and $12 p$, none of our samples showed CNA on the 2 chromosomal arms compared to findings of up to $50 \%$ of samples with $3 q$ gain in a study by He et al. (2003). The difference in frequencies could be due to technical issues (e.g., technology differences, signal cut-off threshold, stromal contamination) or related to the inability to find rare alterations in a small sample size. Such differences also may reflect the distinctness in genomic makeup or exposure to environmental agents among various populations whenever highly recurrent genomic abnormality is considered. The underlying tumor heterogeneity in the studied population as well as among other populations confers pathway redundancy in disease progression and even impacts drug response. 


\begin{tabular}{|c|c|c|c|c|}
\hline CNA & $\begin{array}{c}\text { Current study method: } \\
\text { SNP array }(\mathrm{N}=13), \\
\text { Malaysia }\end{array}$ & $\begin{array}{l}\text { He et al. }(2003) \\
\text { method: CGH } \\
(\mathrm{N}=26) \text {, China }\end{array}$ & $\begin{array}{l}\text { Poeaim et al. }(2005) \\
\text { method: CGH } \\
(\mathrm{N}=40) \text {, Thailand }\end{array}$ & $\begin{array}{l}\text { Nakao et al. }(2004)^{\mathrm{a}} \\
\text { method: aCGH } \\
(\mathrm{N}=125), \text { Spain }\end{array}$ \\
\hline \multicolumn{5}{|l|}{ Gain } \\
\hline $3 q$ & $0.0 \%$ & $50.0 \%$ & $5.0 \%$ & $15.0 \%$ \\
\hline $5 p$ & $23.0 \%$ & $46.0 \%$ & $2.5 \%$ & $10.0 \%$ \\
\hline $7 p$ & $38.5 \%$ & $42.0 \%$ & $17.5 \%$ & $45.0 \%$ \\
\hline $7 q$ & $23.0 \%$ & $42.0 \%$ & $15.0 \%$ & $35.0 \%$ \\
\hline $8 \mathrm{q}$ & $30.8 \%$ & $54.0 \%$ & $25.0 \%$ & $42.0 \%$ \\
\hline $12 p$ & $0.0 \%$ & $38.0 \%$ & $5.0 \%$ & $20.0 \%$ \\
\hline $13 q$ & $46.2 \%$ & $50.0 \%$ & $17.5 \%$ & $30.0 \%$ \\
\hline $19 p$ & $0.0 \%$ & $11.5 \%$ & $20.0 \%$ & $5.0 \%$ \\
\hline $19 q$ & $7.7 \%$ & $11.5 \%$ & $22.5 \%$ & $5.0 \%$ \\
\hline $20 \mathrm{p}$ & $30.8 \%$ & $23.0 \%$ & $10.0 \%$ & $30.0 \%$ \\
\hline $20 \mathrm{q}$ & $61.5 \%$ & $54.0 \%$ & $60.0 \%$ & $65.0 \%$ \\
\hline \multicolumn{5}{|l|}{ Loss } \\
\hline $4 \mathrm{q}$ & $0.0 \%$ & $11.5 \%$ & $20.0 \%$ & $30.0 \%$ \\
\hline $8 p$ & $38.5 \%$ & $23.0 \%$ & $7.5 \%$ & $37.0 \%$ \\
\hline $17 p$ & $38.5 \%$ & $42.0 \%$ & $2.5 \%$ & $46.0 \%$ \\
\hline $18 \mathrm{p}$ & $46.2 \%$ & $46.2 \%$ & $5.0 \%$ & $49.0 \%$ \\
\hline $18 \mathrm{q}$ & $46.2 \%$ & $65.0 \%$ & $25.0 \%$ & $60.0 \%$ \\
\hline
\end{tabular}

${ }^{\text {a} A u t h o r ~ d i d ~ n o t ~ i n d i c a t e ~ w h e t h e r ~ t h e ~ C R C ~ s a m p l e s ~ i n v o l v e d ~ w e r e ~ o f ~ s p o r a d i c ~ c a s e s . ~}$

We next sought alterations that may promote invasiveness and metastatic growth. We compared genomic abnormalities between the early and advanced-stage groups, and the results demonstrated that recurrent gain involving chromosomes $1 \mathrm{q}$ and $5 \mathrm{p}$ occurred only in the advanced-tumor group. One of the advanced tumors had only one gross alteration involving the whole arm of chromosome 1q. It is intriguing that cancer cells with only one gross change can invade and metastasize.

We then looked for genes harbored on the 1q arm that may have functional roles in metastasis. Two genes of interest were transforming growth factor beta 2 (TGFB2) and chromodomain helicase/ATPase DNA binding protein 1-like gene (CHD1L) (Nakagawa et al., 2004; Chen et al., 2010). CHDIL is a recently identified oncogene (Chen et al., 2010) discovered in a study of hepatocellular carcinoma (HCC), which demonstrated increased DNA CN and overexpression in primary HCC samples. Extensive in vivo experimentation has proved that overexpression of $C H D 1 L$ promotes epithelial-mesenchymal transition, an important process in metastasis that enables cancer cells to detach from primary sites and attach to distant organs. Having the same underlying genomic changes as those in HCC, CHDIL in CRC may have the same role.

TGFB2 controls proliferation and differentiation in several cell types (Barton et al., 1988; Attisano and Wrana, 2002). Increased expression of TGFB2 has been associated with cancer-associated stromal fibroblasts in metastatic CRC, providing a conducive microenvironment for tumor growth at foreign sites (Nakagawa et al., 2004). Gain on chromosome 1q has been associated with lymph node metastasis and is even prominent in metastatic CRC tumors (Nakao et al., 2004; Lips et al., 2008). Although a particular tumor may carry other forms of genetic and epigenetic abnormalities (e.g., gene mutation at the nucleotide level and aberrant methylation status) that were beyond the detection capability of the SNP array, findings from these studies shed light on the importance of gain at 1q arm in metastatic advantage. Gain at chromosome 5p, conversely, is rare (De Angelis et al., 2001; Diep et al., 2004; Postma et al., 2009) and suggests different conclusions. 
Diep et al. (2004) have speculated that genes on chromosome 5p are involved in the development of peritoneal carcinomatoses. Conversely, in a study by He et al. (2003) whose majority of the samples were of Dukes' B stage, had a much higher frequency than that seen by other researchers, hence chromosome $5 \mathrm{p}$ could be preferentially altered in the Chinese population in addition to the suggested chromosomes $3 q$ and $12 p$. Our studied samples differ from these 2 studies. No clinical record of the condition of peritoneal seedings was available for the affected samples in our study.

In conclusion, the results of the present study showed that SNP array is a powerful approach for identifying copy-neutral $\mathrm{LOH}$, which remains undetected with CN-based approaches. Oncogenes and tumor suppressor genes harbored on the majority of the chromosomes affected by copy-neutral LOH in this study have yet to be defined, but our results demonstrated a possible role of this event in early CRC progression. Identifying relevant genes and determining whether copy-neutral LOH acts as the second hit in Knudson's theory or is a bridge genomic event to methylation status are daunting tasks yet to be undertaken. Although CRC is a disease that accumulates genomic changes as it progresses, our results also demonstrate that this accumulation does not always occur, at least when we evaluate CNAs and copy-neutral LOH in individual tumors. Using the total number of DNA CNA and copyneutral $\mathrm{LOH}$ events to reflect disease progression may be inappropriate for at least $50 \%$ of the tumors in this study.

\section{ACKNOWLEDGMENTS}

Research supported by the Universiti Sains Malaysia Research University Grant (\#1001/PSK/81202 42). Y.Y. Yam was the recipient of Universiti Sains Malaysia fellowship grant scheme.

\section{REFERENCES}

Andersen CL, Wiuf C, Kruhoffer M, Korsgaard M, et al. (2007). Frequent occurrence of uniparental disomy in colorectal cancer. Carcinogenesis 28: 38-48.

Aragane H, Sakakura C, Nakanishi M, Yasuoka R, et al. (2001). Chromosomal aberrations in colorectal cancers and liver metastases analyzed by comparative genomic hybridization. Int. J. Cancer 94: 623-629.

Ashktorab H, Schaffer AA, Daremipouran M, Smoot DT, et al. (2010). Distinct genetic alterations in colorectal cancer. PLoS One 5: e8879.

Attisano L and Wrana JL (2002). Signal transduction by the TGF- $\beta$ superfamily. Science 296: 1646-1647.

Barton DE, Foellmer BE, Du J, Tamm J, et al. (1988). Chromosomal mapping of genes for transforming growth factors beta 2 and beta 3 in man and mouse: dispersion of TGF-beta gene family. Oncogene Res. 3: 323-331.

Chen L, Chan TH and Guan XY (2010). Chromosome 1q21 amplification and oncogenes in hepatocellular carcinoma. Acta Pharmacol. Sin. 31: 1165-1171.

Choi SW, Lee KJ, Bae YA, Min KO, et al. (2002). Genetic classification of colorectal cancer based on chromosomal loss and microsatellite instability predicts survival. Clin. Cancer Res. 8: 2311-2322.

De Angelis PM, Stokke T, Beigi M, Mjaland O, et al. (2001). Prognostic significance of recurrent chromosomal aberrations detected by comparative genomic hybridization in sporadic colorectal cancer. Int. J. Colorectal Dis. 16: 38-45.

Diep CB, Teixeira MR, Thorstensen L, Wiig JN, et al. (2004). Genome characteristics of primary carcinomas, local recurrences, carcinomatoses, and liver metastases from colorectal cancer patients. Mol. Cancer 3: 6 .

Diep CB, Kleivi K, Ribeiro FR, Teixeira MR, et al. (2006). The order of genetic events associated with colorectal cancer progression inferred from meta-analysis of copy number changes. Genes Chromosomes Cancer 45: 31-41.

Fearon ER and Vogelstein B (1990). A genetic model for colorectal tumorigenesis. Cell 61: 759-767.

He QJ, Zeng WF, Sham JS, Xie D, et al. (2003). Recurrent genetic alterations in 26 colorectal carcinomas and 21 adenomas 
from Chinese patients. Cancer Genet. Cytogenet. 144: 112-118.

Knosel T, Schluns K, Stein U, Schwabe H, et al. (2004). Chromosomal alterations during lymphatic and liver metastasis formation of colorectal cancer. Neoplasia 6: 23-28.

Lagerstedt KK, Kristiansson E, Lonnroth C, Andersson M, et al. (2010). Genes with relevance for early to late progression of colon carcinoma based on combined genomic and transcriptomic information from the same patients. Cancer Inform. 9: 79-91.

Lin CH, Lin JK, Chang SC, Chang YH, et al. (2011). Molecular profile and copy number analysis of sporadic colorectal cancer in Taiwan. J. Biomed. Sci. 18: 36.

Lips EH, van Eijk R, de Graaf EJ, Oosting J, et al. (2008). Integrating chromosomal aberrations and gene expression profiles to dissect rectal tumorigenesis. BMC Cancer 8: 314.

Nakagawa H, Liyanarachchi S, Davuluri RV, Auer H, et al. (2004). Role of cancer-associated stromal fibroblasts in metastatic colon cancer to the liver and their expression profiles. Oncogene 23: 7366-7377.

Nakao K, Mehta KR, Fridlyand J, Moore DH, et al. (2004). High-resolution analysis of DNA copy number alterations in colorectal cancer by array-based comparative genomic hybridization. Carcinogenesis 25: 1345-1357.

Nannya Y, Sanada M, Nakazaki K, Hosoya N, et al. (2005). A robust algorithm for copy number detection using highdensity oligonucleotide single nucleotide polymorphism genotyping arrays. Cancer Res. 65: 6071-6079.

National Cancer Registry (2006). Malaysian Cancer Statistics. Ministry of Health Malaysia, Kuala Lumpur.

Poeaim S, Rerkamnuaychoke B, Jesdapatarakul S and Campiranon A (2005). Chromosome alterations in colorectal cancer in Thai patients. Cancer Genet. Cytogenet. 160: 152-159.

Postma C, Koopman M, Buffart TE, Eijk PP, et al. (2009). DNA copy number profiles of primary tumors as predictors of response to chemotherapy in advanced colorectal cancer. Ann. Oncol. 20: 1048-1056.

Poulogiannis G, Ichimura K, Hamoudi RA, Luo F, et al. (2010). Prognostic relevance of DNA copy number changes in colorectal cancer. J. Pathol. 220: 338-347.

Sheffer M, Bacolod MD, Zuk O, Giardina SF, et al. (2009). Association of survival and disease progression with chromosomal instability: a genomic exploration of colorectal cancer. Proc. Natl. Acad. Sci. U. S. A. 106: 7131-7136.

Staub E, Grone J, Mennerich D, Ropcke S, et al. (2006). A genome-wide map of aberrantly expressed chromosomal islands in colorectal cancer. Mol. Cancer 5: 37.

Stratton MR, Campbell PJ and Futreal PA (2009). The cancer genome. Nature 458: 719-724.

van Puijenbroek M, Middeldorp A, Tops CM, van Eijk R, et al. (2008). Genome-wide copy neutral LOH is infrequent in familial and sporadic microsatellite unstable carcinomas. Fam. Cancer 7: 319-330. 